Commun. Fac. Sci. Univ. Ank. Ser. A1 Math. Stat.

Volume 69, Number 1, Pages 347-353(2020)

DOI: $10.31801 /$ cfsuasmas. 595570

ISSN 1303-5991 E-ISSN 2618-6470

http://communications.science.ankara.edu.tr/index.php?series=A1

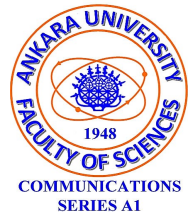

\title{
ON SOME PROPERTIES OF GENERALIZED STRUVE FUNCTION
}

\author{
İBRAHİM AKTAŞ, HALİT ORHAN, AND DORINA RǍDUCANU
}

\begin{abstract}
The main purpose of this investigation is to present some monotonic and log-concavity properties of the generalized Struve function. By using Hadamard product representation of the generalized Struve function, we investigate the sign of this function on some sets. Also, we determine an interval such that the generalized Struve function is decreasing in this interval. Moreover, we show that generalized Struve function is strictly logaritmically concave on some intervals. In addition, we prove that a function related to generalized Struve function is increasing function on $\mathbb{R}$.
\end{abstract}

\section{Introduction and Preliminaries}

In the last three decades many geometric and monotonic properties of some special functions like Bessel, Struve, Lommel, Mittag-Leffler, Wright functions and their generalizations were investigated by many authors. In general, by using the properties of zeros of the special functions many mathematicians studied about univalence, starlikeness, convexity and close-to-convexity of the mentioned functions. In addition, some authors focused on the monotonicity and log-convexity properties of the special functions by using their integral representations and some earlier results on analytic functions. For more information about these investigations the readers are referred to the papers [1, 2, 3, 4, 5, 8, 9, 10, 11] and references therein. Some inequalities which were obtained via above special functions and monotonic properties of these functions are intensively used in engineering sciences, mathematical physics, probability and statistics, and economics. Especially, it is known that the logarithmic concavity and logarithmic convexity properties have an important role in economics. Information on the logarithmic concavity and logarithmic convexity in the economic can be found in [7] and its references, comprehensively. In this study, motivated by the some earlier studies, our main goal is to give some monotonic and log-concavity properties of the generalized Struve functions.

Received by the editors: July 23, 2019; Accepted: November 01, 2019.

2010 Mathematics Subject Classification. Primary 33E50; Secondary 26A48.

Key words and phrases. Gamma function, generalized Struve function, monotonicity, logconcavity.

(C)2020 Ankara University Communications Faculty of Sciences University of Ankara-Series A1 Mathematics and Statistics 
It is well-known that many special functions can be defined by using familiar gamma function. That is why, we want to remember the definition of gamma function. The Euler's gamma or classical gamma function $\Gamma$ is defined by the following improper integral, for $x>0$ :

$$
\Gamma(x)=\int_{0}^{\infty} t^{x-1} e^{-t} d t .
$$

On the other hand, the definition of logarithmic concavity of a function can be given as follow:

Definition 1 ([7]). A function $f$ is said to be log-concave on interval $(a, b)$ if the function $\log f$ is a concave function on $(a, b)$.

The log-concavity of the function $f$ on the interval $(a, b)$ can be shown by using one of the following two conditions:

i. $\frac{f^{\prime}}{f}$ monotone decreasing on $(a, b)$.

ii. $\log f^{\prime \prime}<0$.

Also the following lemma due to Biernacki and Krzyż (see [6]) will be used in order to prove some monotonic properties of the mentioned functions.

Lemma 2. Consider the power series $f(x)=\sum_{n \geq 0} a_{n} x^{n}$ and $g(x)=\sum_{n \geq 0} b_{n} x^{n}$, where $a_{n} \in \mathbb{R}$ and $b_{n}>0$ for all $n \in\{0,1, \ldots\}$, and suppose that both converge on $(-r, r), r>0$. If the sequence $\left\{\frac{a_{n}}{b_{n}}\right\}_{n \geq 0}$ is increasing(decreasing), then the function $x \mapsto\left(\frac{f(x)}{g(x)}\right)$ is also increasing(decreasing) on $(0, r)$.

It is important to note that the above result remains true for the even or odd functions.

\section{Main Results}

In this section, we are going to discuss some properties like monotonicity and log-concavity of the generalized Struve function by using its product representation which is known as Hadamard product or Weierstrassian decomposition. The generalized Struve function has the following series representation (see [12]):

$$
S_{p, b, c, \delta}^{q}(x)=\sum_{n=0}^{\infty} \frac{(-c)^{n}}{n ! \Gamma\left(q n+\frac{p}{\delta}+\frac{b+2}{2}\right)}\left(\frac{x}{2}\right)^{2 n+p+1}
$$

for $q \in \mathbb{N}, p, b, c \in \mathbb{C}$ and $\delta>0$. The author studied some geometric properties such as starlikeness and convexity of generalized Struve function in [12]. Also, the author showed that the zeros of the generalized Struve function are all real. In the same paper, by using Hadamard's theorem an infinite product representation of the generalized Struve function was given as follow (see [12, Lemma 2.1]):

$$
S_{p, b, c, \delta}^{q}(x)=\frac{\left(\frac{x}{2}\right)^{p+1}}{\Gamma\left(\frac{p}{\delta}+\frac{b+2}{2}\right)} \prod_{n \geq 1}\left(1-\frac{x^{2}}{{ }_{q} x_{p, b, c, \delta, n}^{2}}\right),
$$


where ${ }_{q} x_{p, b, c, \delta, n}$ denotes the $n$-th positive zero of the generalized Struve function $S_{p, b, c, \delta}^{q}(x)$.

Theorem 3. Let $b, c, \delta, q$ are positive real numbers,$p>-1$ and ${ }_{q} x_{p, b, c, \delta, n}$ denote the nth positive zero of the generalized Struve function $S_{p, b, c, \delta}^{q}(x)$. In addition, consider the following sets:

$$
\Delta_{1}=\bigcup_{n \geq 1}\left({ }_{q} x_{p, b, c, \delta, 2 n-1, q} x_{p, b, c, \delta, 2 n}\right), \Delta_{2}=\bigcup_{n \geq 1}\left({ }_{q} x_{p, b, c, \delta, 2 n, q} x_{p, b, c, \delta, 2 n+1}\right)
$$

and

$$
\Delta_{3}=\left[0,{ }_{q} x_{p, b, c, \delta, 1}\right) \cup \Delta_{2} .
$$

Then, the generalized Struve function

$$
\Psi_{p, b, c, \delta}^{q}(x)=\left(\frac{2}{x}\right)^{p+1} \Gamma\left(\frac{p}{\delta}+\frac{b+2}{2}\right) S_{p, b, c, \delta}^{q}(x)=\sum_{n=0}^{\infty} \frac{\Gamma\left(\frac{p}{\delta}+\frac{b+2}{2}\right)(-c)^{n}}{n ! \Gamma\left(n q+\frac{p}{\delta}+\frac{b+2}{2}\right)}\left(\frac{x}{2}\right)^{2 n}
$$

satisfies the next properties:

a. the function $x \mapsto \Psi_{p, b, c, \delta}^{q}(x)$ is negative on $\Delta_{1}$ and positive on $\Delta_{3}$,

b. the function $x \mapsto \Psi_{p, b, c, \delta}^{q}(x)$ is decreasing on $\left[0,,_{q} x_{p, b, c, \delta, 1}\right)$,

c. the function $x \mapsto \Psi_{p, b, c, \delta}^{q}(x)$ is strictly log-concave on $\Delta_{3}$.

Proof. a. By considering the infinite product representation of the generalized Struve function $S_{p, b, c, \delta}^{q}(x)$ which is given by 2 , we can easily see that the function $\Psi_{p, b, c, \delta}^{q}(x)$ can be written as the following product representation:

$$
\Psi_{p, b, c, \delta}^{q}(x)=\prod_{n \geq 1}\left(1-\frac{x^{2}}{q^{x_{p, b, c, \delta, n}^{2}}}\right) .
$$

In order to determine the sign of the function $x \mapsto \Psi_{p, b, c, \delta}^{q}(x)$ on the mentioned sets, we rewrite the function $x \mapsto \Psi_{p, b, c, \delta}^{q}(x)$ as

$$
\Psi_{p, b, c, \delta}^{q}(x)=\chi_{n} \tau_{n}
$$

where

$$
\chi_{n}=\prod_{n \geq 1} \frac{{ }_{q} x_{p, b, c, \delta, n}+x}{{ }_{q} x_{p, b, c, \delta, n}^{2}} \text { and } \tau_{n}=\prod_{n \geq 1}\left({ }_{q} x_{p, b, c, \delta, n}-x\right) .
$$

It can be easily seen that $\chi_{n}>0$ for all $x \in[0, \infty)$. On the other hand, since

$$
0<_{q} x_{p, b, c, \delta, 1}<_{q} x_{p, b, c, \delta, 2}<\cdots<_{q} x_{p, b, c, \delta, n}<\cdots
$$

it can be said that, if $x \in\left({ }_{q} x_{p, b, c, \delta, 2 n-1, q} x_{p, b, c, \delta, 2 n}\right)$, then the first $(2 n-1)$ terms of $\tau_{n}$ are strictly negative and remained terms are strictly positive. Also, if $x \in$ $\left({ }_{q} x_{p, b, c, \delta, 2 n},{ }_{q} x_{p, b, c, \delta, 2 n+1}\right)$, then the first $2 n$ terms of $\tau_{n}$ are strictly negative and the rest is strictly positive. In addition, for $x \in\left[0,{ }_{q} x_{p, b, c, \delta, 1}\right)$ all the terms of $\tau_{n}$ are strictly positive. As a consequence, the function $x \mapsto \Psi_{p, b, c, \delta}^{q}(x)$ is negative on $\Delta_{1}$ and it is positive on $\Delta_{3}$. 
b. We know from the previous part of this theorem that the function $x \mapsto \Psi_{p, b, c, \delta}^{q}(x)$ is positive on the interval $\left[0, x_{q} x_{p, b, c, \delta, 1}\right)$. Now, taking logarithmic derivative of (4) implies that

$$
\begin{aligned}
\frac{d}{d x}\left[\log \Psi_{p, b, c, \delta}^{q}(x)\right] & =\frac{\left(\Psi_{p, b, c, \delta}^{q}(x)\right)^{\prime}}{\Psi_{p, b, c, \delta}^{q}(x)} \\
& =\frac{d}{d x}\left[\log \prod_{n \geq 1}\left(1-\frac{x^{2}}{{ }_{q} x_{p, b, c, \delta, n}^{2}}\right)\right] \\
& =\sum_{n=1}^{\infty} \frac{2 x}{x^{2}-{ }_{q} x_{p, b, c, \delta, n}^{2}} .
\end{aligned}
$$

As a result, we get

$$
\left(\Psi_{p, b, c, \delta}^{q}(x)\right)^{\prime}=\Psi_{p, b, c, \delta}^{q}(x) \sum_{n=1}^{\infty} \frac{2 x}{x^{2}-{ }_{q} x_{p, b, c, \delta, n}^{2}}<0
$$

for all $x \in\left[0,{ }_{q} x_{p, b, c, \delta, 1}\right)$. So, the function $x \mapsto \Psi_{p, b, c, \delta}^{q}(x)$ is decreasing on $\left[0,{ }_{q} x_{p, b, c, \delta, 1}\right)$.

c. To show the log-concavity of the function $x \mapsto \Psi_{p, b, c, \delta}^{q}(x)$, it is enough that

$$
\frac{d^{2}}{d x^{2}}\left[\log \Psi_{p, b, c, \delta}^{q}(x)\right]<0
$$

for all $x \in \Delta_{3}$. Now, by using the Hadamard product representation of the function $\Psi_{p, b, c, \delta}^{q}(x)$ which is given by 4 we deduce

$$
\begin{aligned}
\frac{d^{2}}{d x^{2}}\left[\log \Psi_{p, b, c, \delta}^{q}(x)\right] & =\frac{d^{2}}{d x^{2}}\left[\log \prod_{n \geq 1}\left(1-\frac{x^{2}}{{ }_{q}^{2} x_{p, b, c, \delta, n}^{2}}\right)\right] \\
& =\frac{d}{d x}\left[\frac{d}{d x} \sum_{n=1}^{\infty} \log \left(1-\frac{x^{2}}{{ }_{q} x_{p, b, c, \delta, n}^{2}}\right)\right] \\
& =\frac{d}{d x} \sum_{n=1}^{\infty} \frac{-2 x}{{ }_{q} x_{p, b, c, \delta, n}^{2}-x^{2}} \\
& =-2 \sum_{n=1}^{\infty} \frac{{ }_{q} x_{p, b, c, \delta, n}^{2}+x^{2}}{\left({ }_{q} x_{p, b, c, \delta, n}^{2}-x^{2}\right)^{2}} \\
& <0
\end{aligned}
$$

for $x \in \Delta_{3}$. So, the conclusion follows. 
Theorem 4. Let $b, c, \delta, q$ are positive real numbers,$p>-1$ and ${ }_{q} x_{p, b, c, \delta, n}$ denote the nth positive zero of the generalized Struve function $S_{p, b, c, \delta}^{q}(x)$. Then, the function $x \mapsto S_{p, b, c, \delta}^{q}(x)$ is strictly log-concave on $\left(0,,_{q} x_{p, b, c, \delta, 1}\right) \cup \Delta_{2}$.

Proof. By using the fact that the product of two strictly log-concave function is also strictly log-concave, it is possible to prove the log-concavity of the generalized Struve function $S_{p, b, c, \delta}^{q}(x)$ on $\Delta_{3}$. Because of this, we consider the function $S_{p, b, c, \delta}^{q}(x)$ in the following form:

$$
S_{p, b, c, \delta}^{q}(x)=\frac{1}{\Gamma\left(\frac{p}{\delta}+\frac{b+2}{2}\right)}\left(\frac{x}{2}\right)^{p+1} \Psi_{p, b, c, \delta}^{q}(x) .
$$

We known from part c. of Theorem 3 that the generalized Struve function $\Psi_{p, b, c, \delta}^{q}(x)$ is strictly log-concave on $\Delta_{3}$. In addition, since

$$
\frac{d^{2}}{d x^{2}}\left(\log \left(\frac{x}{2}\right)^{p+1}\right)=\frac{d^{2}}{d x^{2}}\left((p+1) \log \left(\frac{x}{2}\right)\right)=-\frac{p+1}{x^{2}}<0
$$

for $p>-1$, the function $x \mapsto\left(\frac{x}{2}\right)^{p+1}$ is strictly log-concave on $(-\infty, 0) \cup(0, \infty)$. As a result, the function $S_{p, b, c, \delta}^{q}(x)$ is strictly log-concave on $\left(0,,_{q} x_{p, b, c, \delta, 1}\right) \cup \Delta_{2}$ as a product of two strictly log-concave functions.

Now, let define the function $x \mapsto h_{p, b, \delta}^{q}(x)$ by putting $c=-1$ in (3). It is easily seen that the function $h_{p, b, \delta}^{q}(x)$ has the following infinite sum representation:

$$
h_{p, b, \delta}^{q}(x)=\sum_{n=0}^{\infty} \frac{\Gamma\left(\frac{p}{\delta}+\frac{b+2}{2}\right)}{n ! 4^{n} \Gamma\left(n q+\frac{p}{\delta}+\frac{b+2}{2}\right)} x^{2 n} .
$$

By using the Lemma 2 we have the following:

Theorem 5. The function

$$
x \mapsto \frac{x\left(h_{p, b, \delta}^{q}(x)\right)^{\prime}}{h_{p, b, \delta}^{q}(x)}
$$

is increasing on $(0, \infty)$ for $p, b, \delta, q \in \mathbb{R}^{+}$.

Proof. By using the infinite sum representation of the function $h_{p, b, \delta}^{q}(x)$ which is given by (5), it can be written that

$$
\frac{x\left(h_{p, b, \delta}^{q}(x)\right)^{\prime}}{h_{p, b, \delta}^{q}(x)}=\frac{\sum_{n=0}^{\infty} A_{n} x^{2 n}}{\sum_{n=0}^{\infty} B_{n} x^{2 n}},
$$

where

$$
A_{n}=\frac{2 n \Gamma\left(\frac{p}{\delta}+\frac{b+2}{2}\right)}{n ! 4^{n} \Gamma\left(n q+\frac{p}{\delta}+\frac{b+2}{2}\right)} \text { and } B_{n}=\frac{\Gamma\left(\frac{p}{\delta}+\frac{b+2}{2}\right)}{n ! 4^{n} \Gamma\left(n q+\frac{p}{\delta}+\frac{b+2}{2}\right)} \text {. }
$$


Cauchy-Hadamard theorem for power series implies that the both series $\sum_{n=0}^{\infty} A_{n} x^{2 n}$ and $\sum_{n=0}^{\infty} B_{n} x^{2 n}$ are convergent for all $x \in \mathbb{R}$, since

$$
\lim _{n \rightarrow \infty}\left|\frac{A_{n}}{A_{n+1}}\right|=\lim _{n \rightarrow \infty}\left|\frac{B_{n}}{B_{n+1}}\right|=\infty .
$$

Moreover, we can say that $A_{n} \in \mathbb{R}$ and $B_{n}>0$ for all $n=0,1,2, \ldots$ On the other hand, if we consider the sequence

$$
\mathcal{H}_{n}=\frac{A_{n}}{B_{n}}=2 n
$$

then we deduce

$$
\frac{\mathcal{H}_{n+1}}{\mathcal{H}_{n}}=\frac{n+1}{n}>1 .
$$

So the sequence $\left\{\mathcal{H}_{n}\right\}_{n \geq 0}$ is increasing. As a result, by applying the Lemma 2 to the function $x \mapsto \frac{x\left(h_{p, b, \delta}^{q}(x)\right)^{\prime}}{h_{p, b, \delta}^{q}(x)}$ the proof is completed.

\section{REFERENCES}

[1] Aktaş, İ. On some properties of hyper-Bessel and related functions, TWMS J. App. Eng. Math., 9(1) (2019), 30-37.

[2] Aktaş, İ. Baricz, Á. and Orhan, H., Bounds for the radii of starlikeness and convexity of some special functions, Turk. J. Math., 42(1) (2018), 211-226.

[3] Aktaş, İ., Baricz, Á. and Singh, S. Geometric and monotonic properties of hyper-Bessel functions, Ramanujan J., doi.org/10.1007/s11139-018-0105-9

[4] Baricz, Á. Geometric properties of generalized Bessel functions, Publ. Math. Debrecen, 73 (2008), 155-178.

[5] Baricz, Á. and Pogány, T. K. Functional inequalities of modified Struve functions, P. Roy. Soc. Edinb. A, 144(5) (2014), 891-904.

[6] Biernacki, M. and Krzyż, J. On the monotonity of certain functionals in the theory of analytic functions, Ann. Univ. Mariae Curie-Sklodowska Sect. A, 9 (1955), 135-147.

[7] Mohtasami Borzadaran, G. R. and Mohtasami Borzadaran, H. A. Log-Concavity property for some well-known distributions, Surv. Math. Appl., 6 (2011), 203-219.

[8] Mondal, S.R. and Akel, M.S. Differential equation and inequalities of the generalized $k$-Bessel functions, J. Inequal. Appl., 2018:175 (2018).

[9] Srivastava, H. M. and Bansal, D. Close-to-convexity of a certain family of $q$-Mittag-Leffler functions, J. Nonlinear Var. Anal., 1 (2017), 61-69.

[10] Srivastava H.M., Murugusundaramoorthy G. and Janani T. Uniformly Starlike Functions and Uniformly Convex Functions Associated with the Struve Function, J. Appl. Computat. Math., 3: 180. doi: 10.4172/2168-9679.1000180

[11] Srivastava, H. M., Murugusundaramoorthy, G. and Sivasubramanian, S. Hypergeometric functions in the parabolic starlike and uniformly convex domains, Integr. Transforms Spec. Funct., 18 (2007), 511-520.

[12] Toklu, E. Radii of starlikeness and convexity of generalized Struve functions, Hacettepe J. Math. Stat., (Accepted). 
Current address: İbrahim Aktaş: Department of Mathematics, Kamil Özdă̆ Science Faculty, Karamanoğlu Mehmetbey University, Yunus Emre Campus, 70100, Karaman-Turkey.

E-mail address: aktasibrahim38@gmail.com

ORCID Address: http://orcid.org/0000-0003-4570-4485

Current address: Halit Orhan: Department of Mathematics, Faculty of Science, Atatürk University, Erzurum-Turkey.

E-mail address: orhanhalit607@gmail.com

ORCID Address: http://orcid.org/0000-0003-3609-5024

Current address: Dorina Răducanu: Faculty of Mathematics and Computer Science, Transilvania University of Braşov, 50091, Iuliu Maniu, 50, Braşov-Romania.

E-mail address: dorinaraducanu@yahoo.com

ORCID Address: http://orcid.org/0000-0003-2348-1874 